\title{
Efeito da nanossílica sobre as propriedades mecânicas de concretos de alta resistência
}

\author{
HIGOR VINÍCIUS STRAPASSON - Mestrando em Engenharia Civil \\ Programa de Pós-Graduação em Engenharia Civil, \\ Universidade Federal de Santa Catarina - UfSC
}

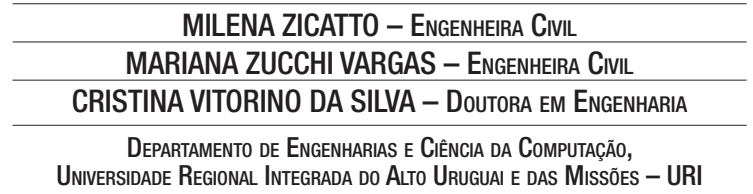

FERNANDA PAVAN LORA - Mestranda em Engenharia CIVIL

Programa de Pós-Graduação Stricto Sensu em Engenharia Civil, Faculdade Meridional - IMED

\section{RESUMO}

O CONCRETO É O MATERIAL MAIS UTILIZADO NA CONSTRUÇÃO DE EDIFICAÇÕES no Brasil. Por INTERMÉdio dos aVAnÇOS teCnOlÓGICOS, INTRODUZIU-SE NO MERCADO O CONCRETO DE ALTA RESISTÊNCIA (CAR) ALIADO À NANOTECNOLOGIA, EM BUSCA POR MELHORIAS NO DESEMPENHO MECÂNICO E NA DURABILIDADE DE ESTRUTURAS. NESTE CONTEXTO, A APLICAÇÃO DE NANOPARTíCULAS DE SÍLICA (NS) EM MATERIAL CIMENTÍCIO SE DESTACA POR INTENSIFICAR SUAS PROPRIEDADES MECÂNICAS, ATUANDO NO PREENCHIMENTO DE VAZIOS E

NA REDUÇÃO DA ZONA DE TRANSIÇÃO, AINDA NO ESTADO FRESCO DA MISTURA. COM O INTENTO DE VERIFICAR A EFICÁCIA DA ADIÇÃO DE NS EM CAR, ESTE ESTUDO PROPÕE AVALIAR O EFEITO DE SUA UTILIZAÇÃO SOBRE AS PROPRIEDADES MECÂNICAS DO CONCRETO, POR MEIO DA ADIÇÃO DE TRÊS TEORES IDEAIS DE NS (0\%, 1,5\% E 3\%) eM RELAÇÃO À MASSA DE CIMENTO PORTLAND. OS RESULTADOS ENCONTRADOS FORAM POSITIVOS EM TODAS AS PROPRIEDADES ESTUDADAS ATRAVÉS DE ANÁLISES DE VARIÂNCIA.

Palavras-chave: concreto de alta resistência, nanotecnologia, nanopartículas de silica.

\section{INTRODUÇÃO}<smiles>C1CCC2CCCCC2CC1</smiles>
concreto é o material de construção mais utilizado pelo homem, sendo um elemento heterogêneo que tem ganhado grande espaço no cenário atual devido a sua versatilidade, resistência e durabilidade. Conciliando a necessidade de fabricação de estruturas mais esbeltas, resistentes e duráveis, com os avanços da tecnologia na construção civil, introduziu-se no mercado os concretos de alto desempenho (CAD) e os concretos de alta resistência (CAR).

Esta inclusão iniciou uma nova era na construção civil, com os concretos de terceira geração com a adição de nanomateriais. Acredita-se que a nanotecnologia seja um dos campos de pesquisa mais promissores para melhorar significativamente o projeto de misturas, bem como o desempenho e a produção de materiais à base de cimento [1].

A revolução da nanotecnologia aprimorou uma grande variedade de produtos, serviços e indústrias, incluindo o setor da construção civil. Uma avaliação abrangente de seus efeitos na saúde humana e ambiental é essencial para o estabelecimento de regulamentos e diretrizes que permitam inúmeros benefícios aos nanomateriais, ao mesmo tempo que forneçam uma proteção adequada aos ecossistemas [2]. 0 campo da nanotecnologia está amadu- recendo rapidamente enquanto área de pesquisa fértil e interdisciplinar a partir da qual novos materiais funcionais e inteligentes são desenvolvidos, como é o caso das nanopartículas de sílica.

A NS é utilizada no concreto para melhorar o seu desempenho, devido à sua ação pozolânica e efeito de preenchimento dos poros entre os grãos de cimento. Além disso, a incorporação de NS no concreto resulta em uma microestrutura mais densa e compacta, redução na absorção de água e melhoria nos parâmetros mecânicos do material, como as resistências à compressão e tração e módulo de elasticidade [3].

Considerando a escassez de pesquisas relacionadas ao desempenho 
Tabela 1 - Matriz experimental do estudo

\begin{tabular}{|c|c|c|c|c|c|}
\hline & \multicolumn{5}{c|}{ Combinações } \\
\hline Fatores controláveis & A & B & C & Número de CP's \\
\hline Teor de adição de NS (\%) & 0,0 & 1,5 & 3 & $\begin{array}{c}\text { (por combinação) } \\
\text { a serem ensaiados }\end{array}$ \\
\hline Variáveis resposta & \multicolumn{4}{c|}{ Idades (dias) } \\
\hline Absorção capilar & 28 & 4 & 0 & $0,37 \pm 0,03$ \\
\hline Módulo de elasticidade & 28 & 4 & 0 & $0,37 \pm 0,03$ \\
\hline Resistência à compressão uniaxial & 28 & 4 & 0 & $0,37 \pm 0,03$ \\
\hline Resistência à tração por compressão uniaxial & 28 & 4 & 0 & $0,37 \pm 0,03$ \\
\hline Resistência à tração na flexão & 28 & 2 & 0 & $0,37 \pm 0,03$ \\
\hline Fonte: Autores (2021) & & & & \\
\hline
\end{tabular}

mecânico do CAR com a inserção de NS, este estudo busca analisar a influência das adições de 1,5\% e 3\% de NS em suspensão coloidal no referido material, em comparação às amostras do concreto sem qualquer adição, verificando as características que cada teor adicionado exerce sobre o mesmo.

\section{METODOLOGIA}

Para compreender como a NS atua nas matrizes do CAR, definiu-se os teores ótimos de adição da mesma (1,5\% e 3\%) e, posteriormente, determinou-se a quantidade de corpos de provas (CP's) a serem moldados para cada ensaio proposto, de acordo com matriz experimental do estudo, conforme mostra a Tabela 1.

A caracterização dos materiais utilizados foi realizada em conformidade com as normativas vigentes, obtendo-se os seguintes resultados:

a) cimento CPV-ARI, com finura por meio da peneira $75 \mu \mathrm{m}$ igual a $1,91 \%$ e massa específica igual a $3,04 \mathrm{~g} / \mathrm{cm}^{3}$

b) agregado miúdo e graúdo, com massa unitária respectivamente, igual a $1,54 \mathrm{~g} / \mathrm{cm}^{3}$ e $1,60 \mathrm{~g} / \mathrm{cm}^{3}$, massa específica igual a 2,92 g/ $/ \mathrm{cm}^{3}$ e 2,84 g/ $\mathrm{cm}^{3}$, absorção de água igual a 0,60\% e 0,78\%, módulo de finura igual a 1,62 e 7,01, dimensão máxima característica (DMC) equivalente a 2,36 $\mathrm{mm}$ e $19 \mathrm{~mm}$;

c) nanopartículas de sílica compostas por $15 \%$ de sólidos, com massa específica e pH igual a $1,1 \mathrm{~g} / \mathrm{cm}^{3}$ e 9,5 respectivamente, sob forma de solução coloidal aquosa de sílica amorfa; d) sílica ativa, com massa específica tituição considerando 10\% do volume de cimento;

e) aditivo superplastificante à base de policarboxilicatos, livre de cloretos e com massa específica igual a $1,1 \mathrm{~g} / \mathrm{cm}^{3}$.

O método de dosagem experimental adotado para a produção do CAR $\left(f_{c k}\right.$ de $55 \mathrm{MPa}$ ) foi o desenvolvido pelo igual a $2,20 \mathrm{~g} / \mathrm{cm}^{3}$ e teor de subs-
Instituto de Pesquisas Tecnológicas do estado de São Paulo (IPT) modificado por Cremonini (2001) [4]. Os resultados são evidenciados na Tabela 2.

De acordo com a Tabela 2, os valores de resistência obtidos para os três traços apresentaram resistências maiores que o fck inicial de $55 \mathrm{MPa}$. Assim, optou-se por utilizar o traço 1:5 para a confecção dos CP's, por apresentar um menor consumo de cimento.

Os ensaios foram elaborados no Laboratório de Preparo de Amostras e de Técnicas Construtivas (LAPATEC), localizado no Campus 2 da Universidade Regional Integrada do Alto Uruguai e das Missões - URI Campus de Erechim.

Dentre a instrumentação utilizada, conforme mostra a Figura 1, foram empregados diferentes tipos de fôrmas metálicas para a moldagem das amostras de concreto. Para os ensaios de absorção de água por capilaridade e resistência à compressão uniaxial, os CP's foram produzidos em fôrmas com dimensão $\varnothing 100$ × 200 mm; já para os ensaios de resistência à tração na flexão, as dimensões das fôrmas utiliza-

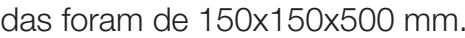

Após a moldagem, os CP's foram adensados em mesa vibratória e cobertos por 24 horas com uma lona plástica para evitar a perda de água da mistura para o ambiente externo. Em seguida,
Tabela 2 - Traços utilizados na dosagem dos concretos

\begin{tabular}{|c|c|c|c|c|c|c|c|c|c|c|c|}
\hline \multirow[b]{2}{*}{ m } & \multirow[b]{2}{*}{$\begin{array}{c}\alpha \\
(\%)\end{array}$} & \multicolumn{4}{|c|}{ Traço unitário } & \multirow[b]{2}{*}{$\begin{array}{l}\text { Slump } \\
(\mathrm{mm})\end{array}$} & \multirow[b]{2}{*}{$\begin{array}{c}\text { Aditivo } \\
(\%)\end{array}$} & \multirow{2}{*}{$\begin{array}{c}\text { Consumo } \\
\text { de } \\
\text { cimento } \\
\left(\mathrm{kg} / \mathrm{m}^{3}\right)\end{array}$} & \multirow[b]{2}{*}{$\begin{array}{c}\mathrm{H} \\
(\%)\end{array}$} & \multirow[b]{2}{*}{$\begin{array}{c}\text { Resistência } \\
\mathrm{F}_{\mathrm{cj}} \\
(\mathrm{MPa})\end{array}$} & \multirow[b]{2}{*}{$\mathrm{a} / \mathrm{c}$} \\
\hline & & c & a & p & sa & & & & & & \\
\hline 2 & - & 1,0 & 0,5 & 1,5 & 0,1 & 150 & 1,387 & 791,0 & 0,065 & 68,70 & 0,20 \\
\hline 3,5 & 0,5 & 1,0 & 1,25 & 2,25 & 0,1 & 130 & 0,932 & 519,2 & 0,065 & 63,90 & 0,29 \\
\hline 5 & - & 1,0 & 2,00 & 3,00 & 0,1 & 150 & 0,856 & 386,5 & 0,065 & 65,26 & 0,39 \\
\hline
\end{tabular}




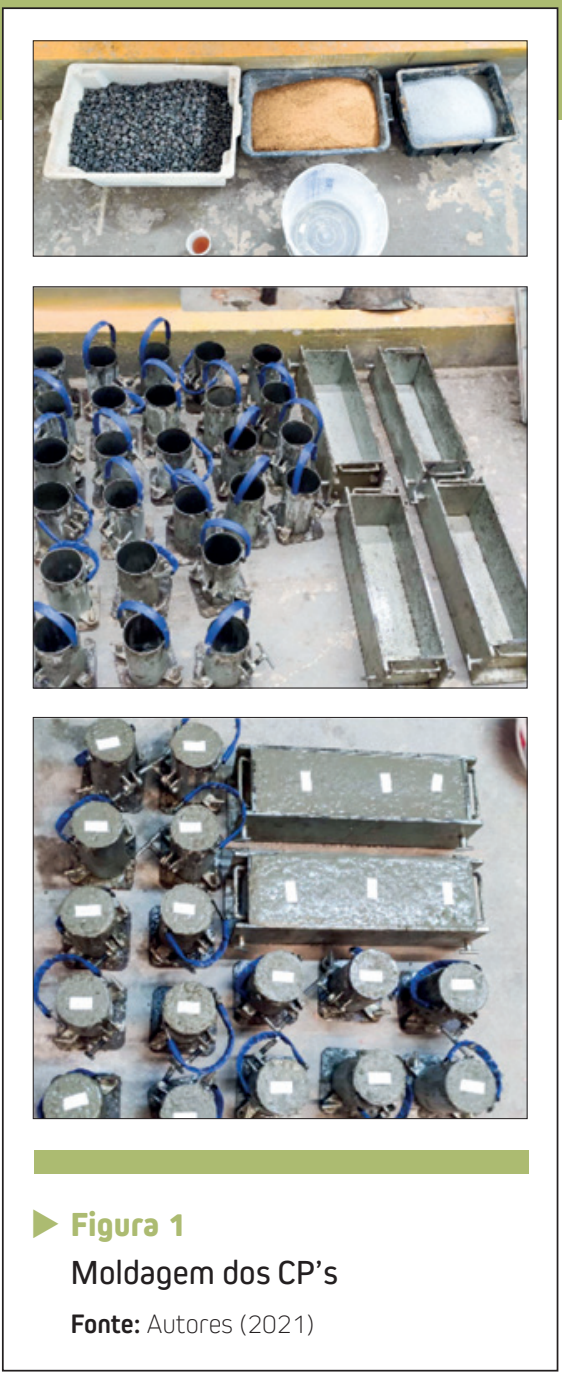

os CP's foram desmoldados e mantidos em câmara úmida (UR $\geq 95 \%$ e temperatura de $23^{\circ} \mathrm{C} \pm 2$ ), para posterior realização dos ensaios.

\section{RESULTADOS E DISCUSSÕES}

Para a análise dos resultados, eliminou-se os dados hipotéticos e, em seguida, a análise dos dados foi efetuada a partir do método de variância (ANOVA) de uma via (One Way), com o auxílio do software Statistica. A Tabela 3 mostra um resumo dos resultados encontrados para cada ensaio realizado, onde a análise e a interpretação dos resultados será evidenciada através dos tópicos abaixo.

\section{I Resistência à compressão uniaxial}

Analisando a Tabela 3, é possí- vel verificar que a adição de 1,5\% de NS não influenciou significativamente o desempenho do CAR quanto a sua resistência à compressão uniaxial, diferentemente da adição de 3\% do nanomaterial, que culminou em resultados menores de resistência quando em comparação as amostras de referência.

De modo geral, o aumento da resistência à compressão mediante a inserção de um nanomaterial pode relacionar-se ao efeito filler e a criação de pontos de nucleação nas misturas cimentícias. Neste caso, como o aumento de resistência não foi significativo nas amostras com $1,5 \%$ de NS, então pode-se concluir pela atuação do efeito fíler. Além disso, acredita-se que a não realização da dispersão do material tenha promovido a agregação das partículas de sílica, possibilitando uma instabilidade na dispersão coloidal e evidenciando reduções na resistência à compressão [5].

\subsection{Resistência à tração por compressão diametral}

Por meio da análise da Tabela 3, verifica-se que o efeito da adição de NS não foi significativo na variável de resposta em ambas as porcentagens de adição. Pode-se concluir pela necessidade de maiores porcentagens de adição de NS sobre o CAR, para a promoção de um aumento de resistência à compressão diametral, além de períodos maiores de cura.

De acordo com o estudo realizado por Silva Junior (2017), a resistência à tração por compressão diametral dos concretos cresce com o aumento do teor de adição de nanossílica [6]. A adição de $2 \%$ de NS, combinada com o teor de água ótimo, apresenta um bom desempenho relacionado com a resistência à tração, justificando-se que a adição de 1,5\% desta pesquisa não apresentou tal efeito significativo. Além disso, aconselha-se verificar as propriedades em idades mais avançadas de cura (por exemplo aos 90 e 180 dias) como forma de identificar aumentos na resistência, conforme identificado pelo autor mencionado acima.

\subsection{Resistência à tração na flexão}

Por meio da Tabela 3, percebe-se que o efeito da adição de NS não foi significativo na variável de resposta em ambas as porcentagens. Verifica-se também que as amostras com adições promoveram respectivamente aumentos de $6,48 \%$ e 9,88\% na resistência à tração na flexão do CAR quando comparadas às amostras de concreto sem adição. Em ambos os teores de adição de NS, observou-se um ganho linear de resistência devido à grande capacidade que a matriz do compósito, com a contribuição das nanopartículas de sílica de resistir aos efeitos de tração.

As nanopartículas de sílica promovem uma atuação física, ocupando os espaços intersticiais existentes entre os grãos de cimento, desempenhando o papel de fíler. Como resultado, uma maior densificação da matriz de cimento será obtida e, por conseguinte, uma maior resistência e durabilidade de pastas, argamassas ou concretos [7].

\subsection{Módulo de elasticidade}

Analisando a Tabela 3, verificou-se um aumento de $5,50 \%$ ou $2,15 \mathrm{GPa}$ no concreto com adição de $1,5 \%$ de 
NS em relação ao concreto referência, não apresentando efeito significativo na variável de resposta. Já, a adição de 3\% de NS obteve uma influência significativa e positiva da incorporação de NS nos compósitos, onde a adição obteve um acréscimo de $10,7 \%$ em relação ao concreto referência. Diante disso, observou-se um aumento contínuo no módulo de elasticidade em ambas as porcentagens devido à grande flexibilidade transversal que as nanopartículas de sílica possuem em resistir aos efeitos.

Segundo Mendes (2016), o módulo de elasticidade pouco se altera em função da quantidade de nanossílica, assim como a densidade do material. A presença de agregados e a interface matriz-agregado são fatores determinantes no módulo de elasticidade do concreto, que atribuem a nanossílica um efeito diferente do observado em matrizes cimentícias, sendo um destes fatores que contribuíram para os baixos ganhos no módulo de elasticidade desta pesquisa [8].

\subsection{Absorção de água por capilaridade}

Através da análise da Tabela 3, observa-se que tanto o concreto sem adição quanto o concreto com adição de NS apresentaram um comportamento linear de crescimento. Ainda, verificam-se aumentos de 16,8\% e 10,9\% na absorção média capilar dos concretos com adição de NS, sem a promoção de efeitos significativos nas variáveis de resposta.

Devido ao tamanho reduzido das nanopartículas de sílica, as mesmas acabam preenchendo os vazios existentes na mistura, propiciando um maior

\begin{tabular}{|c|c|c|c|c|c|}
\hline $\begin{array}{l}\text { Teor de } \\
\text { adição de NS } \\
(\%)\end{array}$ & $\begin{array}{l}\text { Média } \\
\text { (MPa) }\end{array}$ & $\begin{array}{l}\text { Desvio } \\
\text { padrão } \\
\text { (MPa) }\end{array}$ & $\begin{array}{l}\text { Coeficiente } \\
\text { de variação } \\
\text { (MPa) }\end{array}$ & p & Significância \\
\hline \multicolumn{6}{|c|}{ Resistência à compressão uniaxial } \\
\hline 0,0 & 75,16 & 3,42 & 4,55 & - & - \\
\hline 1,5 & 75,34 & 2,90 & 3,85 & 0,948853 & NS \\
\hline 3,0 & 59,22 & 0,76 & 1,28 & 0,008502 & S \\
\hline \multicolumn{6}{|c|}{ Resistência à tração por compressão diametral } \\
\hline 0,0 & 5,11 & 0,56 & 10,94 & - & - \\
\hline 1,5 & 5,36 & 0,46 & 8,67 & 0,580335 & NS \\
\hline 3,0 & 5,00 & 0,16 & 3,11 & 0,821823 & NS \\
\hline \multicolumn{6}{|c|}{ Resistência à tração na flexão } \\
\hline 0,0 & 6,17 & 0,27 & 4,35 & - & - \\
\hline 1,5 & 6,57 & 0,40 & 6,03 & 0,358665 & NS \\
\hline 3,0 & 6,78 & 0,04 & 0,63 & 0,086699 & NS \\
\hline \multicolumn{6}{|c|}{ Módulo de elasticidade } \\
\hline 0,0 & 39,13 & 1,17 & 2,99 & - & - \\
\hline 1,5 & 41,28 & 1,14 & 2,77 & 0,059596 & NS \\
\hline 3,0 & 43,35 & 0,07 & 0,16 & 0,006641 & S \\
\hline \multicolumn{6}{|c|}{ Absorção de água por capilaridade } \\
\hline 0,0 & $0,19523^{\star}$ & 0,01 & 7,53 & - & - \\
\hline 1,5 & $0,22794^{\star}$ & 0,02 & 7,90 & 0,569904 & NS \\
\hline 3,0 & $0,21645^{\star}$ & 0,03 & 15,49 & 0,558836 & NS \\
\hline $\begin{array}{l}\text { p: nível de signific } \\
\text { absorção após } 72 \\
\text { Fonte: Autores (2 }\end{array}$ & valor signif & alor $n$ & tivo. Se $p<5 \%$ & o significativ & es médios de \\
\hline
\end{tabular}

empacotamento da matriz cimentícia e diminuindo significativamente o diâmetro dos poros. No CAR, a água permeia pela matriz e também pela interface matriz-agregado; pode-se concluir que é provável que os parâmetros relacionados a permeabilidade dos concretos, principalmente em relação a interface matriz-agregado, são influenciados pela adição de NS, explicando, em parte, os resultados aqui obtidos.

\subsection{Análise comparativa dos resultados obtidos para as variáveis de resposta}

A Tabela 4 contém um resumo comparativo dos resultados obtidos para as variáveis de resposta, analisadas em porcentagem, com relação ao concreto referência. A Figura 2 mostra um compilado de todos os ensaios.
Conforme a Tabela 4, a incorporação de $1,5 \%$ de NS proporcionou um leve crescimento de 1,08\% na resistência à compressão uniaxial e um aumento de 4,89\% na resistência à tração por compressão diametral. Nos ensaios de resistência à tração na flexão e módulo de elasticidade, ocorreram acréscimos de 6,5\% e 5,5\%, respectivamente, em comparação ao concreto referência. Em ambos os ensaios supracitados, os aumentos de resistência não foram expressivos.

A adição de 3\% de NS promoveu reduções de $21,2 \%$ na resistência à compressão uniaxial e 1,96\% na resistência à tração por compressão diametral. Em contrapartida, os ensaios de resistência à tração na flexão e módulo de elasticidade proporcionaram acréscimos de 9,9\% e 10,9\%, em relação ao concreto referência. 
Tabela 4 - Resumo comparativo dos resultados obtidos para as variáveis de resposta

\begin{tabular}{|c|c|c|c|c|c|}
\hline \multicolumn{6}{|c|}{ Variáveis de resposta } \\
\hline $\begin{array}{c}\text { Teor de } \\
\text { adição (\%) }\end{array}$ & $\begin{array}{c}\text { Resistência à } \\
\text { compressão } \\
\text { uniaxial }\end{array}$ & $\begin{array}{c}\text { Resistência } \\
\text { à traçãa por } \\
\text { compressão } \\
\text { diametral }\end{array}$ & $\begin{array}{l}\text { Resistência } \\
\text { à tração na } \\
\text { flexão }\end{array}$ & $\begin{array}{l}\text { Módulo de } \\
\text { elasticidade }\end{array}$ & $\begin{array}{l}\text { Absorção } \\
\text { de água por } \\
\text { capilaridade }\end{array}$ \\
\hline 0,0 & Referência & Referência & Referência & Referência & Referência \\
\hline 1,5 & $\uparrow 1,08 \%$ & $\uparrow 4,89 \%$ & $\uparrow 6,48 \%$ & $\uparrow 5,50 \%$ & $\uparrow 16,75 \%$ \\
\hline 3,0 & $\downarrow 21,21 \%$ & $\downarrow 1,96 \%$ & $\uparrow 9,88 \%$ & $\uparrow 9,25 \%$ & $\uparrow 10,87 \%$ \\
\hline \multicolumn{6}{|c|}{ Fonte: Autores (2021) } \\
\hline
\end{tabular}

De modo geral, as adições de NS indicaram que o desempenho do concreto é melhorado, acreditando-se que os acréscimos estão relacionados ao efeito fíller e a reação pozolânica da matriz cimentícia. $O$ tamanho reduzido das nanopartículas fornece uma área de superfície maior, promovendo uma aceleração da taxa de hidratação e das reações pozolânicas [9].

\section{CONSIDERAÇÕES FINAIS}

Com base na análise estatística dos resultados, verificou-se que a adição de NS influenciou significativamente apenas as amostras de CAR contendo 3\% de NS, mediante o ensaio de módulo de elasticidade, em comparação ao concreto referência (sem nenhuma adição).

Dentre todas as variáveis de resposta, a absorção média de água por capilaridade apresentou o maior quantitativo em relação ao concreto de referência $(16,8 \%)$, constatando-se como um efeito desfavorável na zona matriz-agregado, o que favorece o acesso de agentes agressivos e, posteriormente, a degradação do material. A água é o agente responsável por transportar substâncias que podem causar danos ao concreto e, por isso, é necessário reduzir o seu fluxo para o interior do material, assim evitar danos ao mesmo a partir da sua microestrutura. Consideramos necessário desenvolver uma nova fase de estudos focando-se na otimização da dispersão/funcionalização das nanopartículas de sílica que deverá envolver inclusive a caracterização da NS por técnicas químicas apropriadas como a espectrometria de Raman.

Acredita-se também que os pequenos acréscimos de quantitativos obtidos nas variáveis de resposta devem-se a não utilização do método de dispersão/funcionalização das na- nopartículas de sílica, mesmo o nanomaterial já estando disperso em meio aquoso. Conforme cita a literatura, a dispersão do material é necessária, já que as partículas tendem a se aglomerar, devido a influência de forças de Van Der Waals, que reduzem as superfícies livres das moléculas e resultam na redução do seu desempenho dentro da matriz cimentícia [10].

De modo geral, as adições de 1,5\% e 3\% de NS não apresentaram efeitos satisfatórios no CAR. Esperava-se que a adição de NS promovesse um aumento na resistência do concreto, tornando a zona de transição mais compacta e coesa. Conclui-se, então, a necessidade de redução da adição na ordem de $1 \%$ de NS para que ocorram significativas melhorias nas propriedades

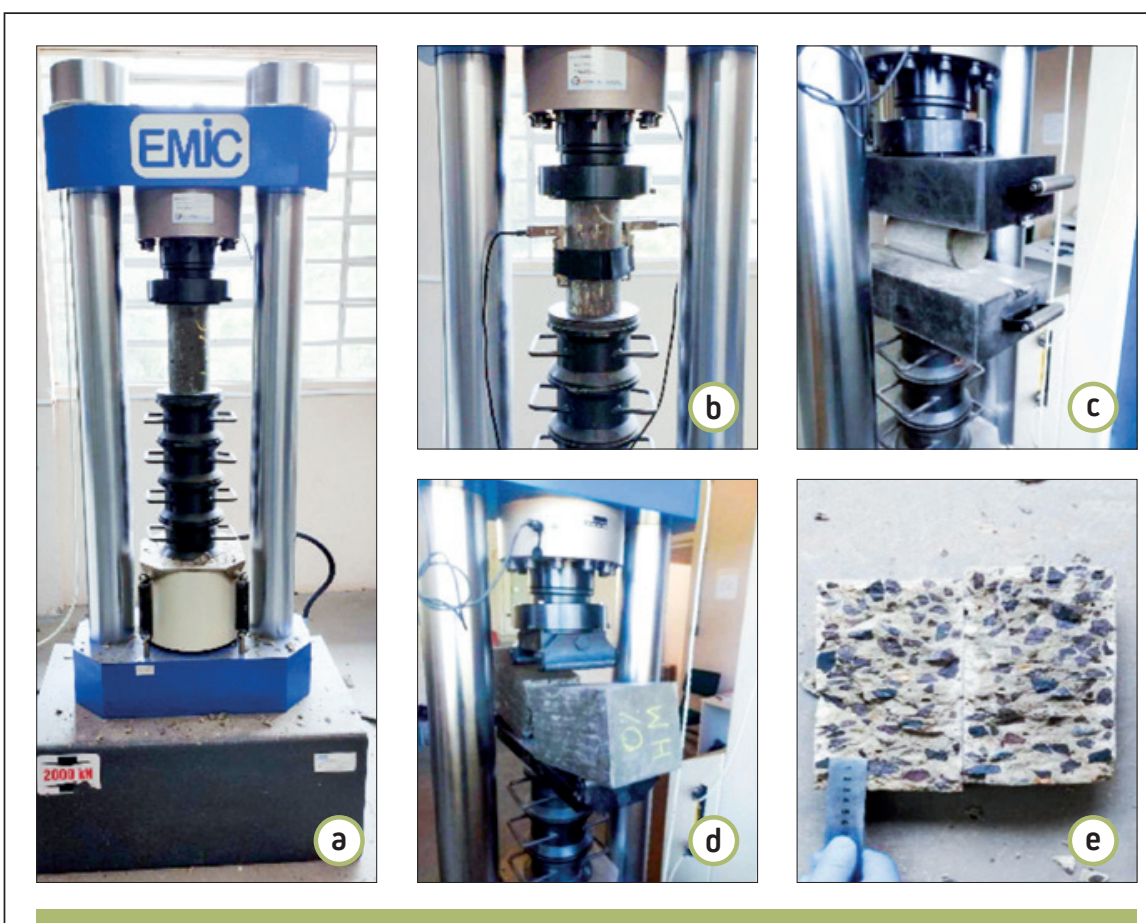

Figura 2

Ensaios realizados: a) Resistência à compressão uniaxial; b) Módulo de elasticidade; c) Resistência à tração por compressão diametral; d) Resistência à tração na flexão; e) Absorção capilar

Fonte: Autores (2021) 
mecânicas do material, tanto no estado fresco como endurecido.

É importante salientar que, atualmente, os maiores desafios encontrados para a inserção das nanopartículas de sillica na construção civil é a sua escassa produção industrial e os elevados custos relativos a mesma. Desta forma, demonstra-se necessário o crescimento da nanotecnologia no setor da construção civil, em conjunto com investimentos notórios para o seu desenvolvimento, permitindo antever um sucesso crescente da aplicação desta tecnologia a médio e a longo prazo.

\section{REFERENCIAS BIBLIOGRÁFICAS}

[1] SAID, A. M. et al. Properties of concrete incorporating nano-silica. Construction and Building Materials. v. 36, p.838-844, nov. 2016. Elsevier.

[2] LEE, J.; MAHENDRA, S.; ALVAREZ, P. J. J. Nanomaterials in the Construction Industry: a review of their applications and environmental health and safety considerations. Acs Nano, v. 4, n. 7, p. 3580-3590, jul. 2010. American Chemical Society (ACS).

[3] SINGH, L. P. et al. Beneficial role of nanosilica in cement based materials - A review. Construction and Building Materials. v. 47, p.1069-1077, out. 2013. Elsevier.

[4] CREMONINI, R. A. et al. Desenvolvimento de um método de dosagem de concretos de alta resistência com baixo consumo de cimento, IBRACON, 2001.

[5] SOUSA, I. P. S. Obtenção da nanossílica funcionalizada visando o uso como aditivo em misturas cimentícias. 2017. 89 f. Dissertação, Universidade Federal de Goias, Goiânia/GO, 2017.

[6] SILVA JUNIOR, P. F. Estudo da estabilidade dimensional de concretos de alta resistência com adição de polímero superabsorvente e nanopartículas de sílica. 2017. 368 f. Tese, Programa de Pós-graduação em Engenharia Civil e Ambiental, Universidade de Brasília, Brasília, 2017.

[7] SENFF, L.; HOTZA, D.; REPETTE, W. L. Comportamento reológico de pastas de cimento com adição de sílica ativa, nanossílica e dispersante policarboxílico. Revista Matéria, v. 15, nº 1, p. 12-20, 2010.

[8] MENDES, T. M. Efeito da nanossílica em matrizes cimentícias de ultra alta resistência. 2016. 113 f. Tese, Programa de pós-graduação em Engenharia Civil Universidade Federal de Santa Catharina, Florianópolis, 2016.

[9] SAID, A. M. et al. Properties of concrete incorporating nano-silica. Construction And Building Materials, v. 36, p. 838-844, nov. 2012. Elsevier BV.

[10] YANG, R. Y. et al. Agglomeration of fine particles subjected to centripetal compaction. Powder Technology, v. 184, 2008.

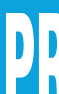 (illith}

\section{Macrofibras poliméricas para concreto destinado a aplicaçoes estruturais:' definições, especificações e conformidade}

Elaborada pelo CT 303 - Comitê Técnico IBRACON/ABECE sobre Uso de Materiais não Convencionais para Estruturas de Concreto, Fibras e Concreto Reforçado com Fibras, a Prática Recomendada especifica os requisitos técnicos das macrofibras poliméricas para uso em concreto estrutural.

A Prática Recomendada abrange macrofibras para uso em todos os tipos de concreto, incluindo concreto projetado, para pavimentos, pré-moldados, moldados no local e concretos de reparo.

\section{A Q U I S I C Ã O}

www.ibracon.org.br (Loja Virtual)

\section{DADOS TÉCNICOS}

ISBN: 978-85-98576-29-9

Edição: $1^{\mathrm{a}}$ edição

Formato: eletrônico

Páginas: 37

Acabamento: digital

Ano da publicação: 2017

Coordenador: Eng. Marco Antonio Carnio
PRÁTICA RECOMENDADA IBRACON/ABECE MACROFIBRAS POLIMÉRICAS PARA CONCRETO DESTINADO A APLICAÇÕES ESTRUTURAIS

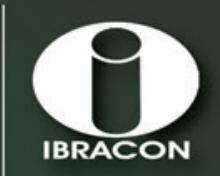

Patrocínio 